\title{
ANÁLISIS DE LOS FACTORES SUBYACENTES CONSTRUCTORES DE VULNERABILIDADES ANTE RIESGO DE DESASTRES EN EL ESTADO DE MÉXICO
}

\author{
JOSÉ EMILIO BARÓ SUÁREZ
}

\author{
Universidad Autónoma del Estado de México
}

Armando Arriaga Rivera

Sociedad Hijos de Calimaya, A. C.

CARlos díaz Delgado

Universidad Autónoma del Estado de México

\begin{abstract}
Resumen: El objetivo de este trabajo es realizar un análisis de los factores subyacentes constructores de vulnerabilidades ante riesgo de desastres en el Estado de México, enfocado principalmente en las inundaciones, por ser el riesgo extensivo con mayores impactos y daños al ambiente y a la sociedad. Estos factores subyacentes que se mencionan durante el trabajo también son válidos para los riesgos geológicos. El trabajo considera los pocos estudios realizados sobre atlas de riesgo y de ordenamiento ecológico en los municipios del Estado de México, porque no está cubierto todo el estado.
\end{abstract}

Palabras clave: factores subyacentes, atlas de riesgo, ordenamientos ecológicos, riesgos socionaturales, Estado de México.

\begin{abstract}
The objective of this work is to carry out an analysis of the underlying factors that build vulnerability to disaster risk in the State of Mexico, focused mainly on floods, as it is the extensive risk with greater impacts and damage to the environment and society. These underlying factors that are mentioned during work are also valid for geological hazards. The work considers the few studies carried out on atlases of risk and ecological order in the municipalities of the State of Mexico, because the entire state is not covered.

Keywords: Underlying Factors, Risk Atlases, Ecological Systems, Socionatural Risks and the State of Mexico.
\end{abstract}

\section{Introducción}

Según el Global Assessment Report (GAR, 2009) los factores subyacentes constructores de vulnerabilidades ante el riego de desastres son "aquellos procesos, tanto físicos como sociales, que contribuyen, impulsan, conducen o determinan de forma importante a la construcción, creación o existencia de condiciones de riesgo de desastres en la sociedad". El objetivo de este trabajo es efectuar un análisis de estos factores subyacentes por lo que 
Análisis de los factores subyacentes constructores de vulnerabilidades ante riesgo de desastres en el Estado de México

se analizan las condicionantes socioeconómicas y de cumplimiento de las políticas públicas en materia de Protección Civil en varios municipios del Estado de México para con ello contribuir en la mitigación y reducción de los desastres en los asentamientos humanos.

Partiendo de lo anteriormente expuesto, el estudio tiene gran importancia para el Estado ya que, a partir del mismo y en conjunto con otras investigaciones enfocadas en este tema, podrá plantear una serie de medidas estructurales y no estructurales, además de identificar y focalizar aquellos recursos destinados a reducir el riesgo y prevenir los desastres.

Este trabajo se enfoca básicamente en las inundaciones como el riesgo extensivo que mayores impactos y daños causa en el ambiente y a la sociedad mexiquense. Aunque los factores subyacentes que se mencionan también son válidos para otros escenarios de riesgos geológicos (sismos, volcanes y movimientos gravitacionales).

Se considera el Estado de México como el estado del país que más impacto recibe por inundaciones y mayores daños sufre, ya que es el más poblado y el que mayor infraestructura y viviendas (mayor valor de los bienes) tiene expuestas ante estos fenómenos.

\section{Metodología}

Este trabajo consta de cuatro apartados: 1) identificación de factores subyacentes; 2) desglose de factores subyacentes del riesgo (para evitar la generación y acumulación del riesgo); 3) causas o factores subyacentes preponderantes en el Estado de México que son constructores de escenarios de riesgo por inundación; y 4) propuestas de medidas de políticas públicas para la reducción de la vulnerabilidad ante riesgo de desastres en el Estado de México.

\section{Resultados y discusión}

\subsection{Identificación de factores subyacentes}

Para la identificación de los factores subyacentes se llevó a cabo una búsqueda de información a nivel municipal para su análisis, revisión e integración. En los cuadros 1 y 2 se detallan los municipios del Estado de México que se encuentran afectados por fenómenos hidrometeorológicos y geológicos (cuadro 1 y mapa 1), así como los municipios con mayor incidencia de inundaciones, población afectada y daños (cuadro 2). Como se puede observar en el cuadro 2, los municipios con mayores incidencias de inundaciones durante el período del 2002 al 2016, según datos de la CAEM (2017) publicados en los Atlas de Inundaciones, son: 
José Emilio Baró Suárez - Armando Arriaga Rivera - Carlos Díaz Delgado

- Atizapán de Zaragoza

- Chimalhuacán

- Cuautitlán Izcalli

- Ecatepec de Morelos

- Nezahualcóyotl

- Tlalnepantla de Baz

- Tultitlán

De entre estos municipios, hay que destacar el municipio de Ecatepec de Morelos, en el Valle de México. Este está afectado por varios tipos de fenómenos hidrometeorológicos que pueden provocar daños materiales de importancia ya que sobre todo está expuesto inundaciones y a lluvias extraordinarias. Acontecimientos como las inundaciones de 2011-2012, constituyen los ejemplos más recientes que ponen de manifiesto la gravedad de las consecuencias de esta clase de fenómenos. Las precipitaciones pluviales han provocado que el Ecatepec se encuentre entre los municipios con mayores problemas por inundaciones en el centro del país. Este tipo de riesgos, además de la emergencia humanitaria que desencadena, acarrea problemas sociales, económicos y de salud (H. Ayuntamiento de Ecatepec de Morelos, s/f: 185).

En los cuadros 3 y 4 se enumeran, por año de publicación, los municipios del Estado de México que cuentan con atlas de riesgo o algún instrumento de ordenamiento territorial, Los municipios que cuentan con este tipo de instrumentos son 65 municipios, lo que representan el 52\% del total de los 125 municipios de la entidad.

\begin{tabular}{clc}
\hline No. & \multicolumn{1}{c}{ Municipios } & Fenómeno \\
\hline 1 & Atizapán de Zaragoza & Inundaciones \\
2 & Chimalhuacán & Inundaciones \\
3 & Cuautitlán Izcalli & Inundaciones \\
4 & Ecatepec de Morelos & Inundaciones \\
5 & Naucalpan & Inundaciones y Remoción en masa \\
6 & Nezahualcóyotl & Inundaciones \\
7 & San Mateo Atenco & Inundaciones \\
8 & Tenancingo & Sismos y Remoción en masa \\
9 & Tenango del Valle & Sismos y Remoción en masa \\
10 & Tlalnepantla de Baz & Inundaciones \\
11 & Texcoco & Inundaciones \\
12 & Toluca & Inundaciones, Remoción en masa y agrietamientos \\
13 & Tultitlán & Inundaciones \\
\hline
\end{tabular}

Cuadro 1. Relación de municipios con mayor incidencia de fenómenos hidrometeorológicos y geológicos en el Estado de México (Fuente: Elaboración propia con base en el Atlas de Riesgo del Estado de México, municipales y el Atlas de Riesgo de Inundación de la Comisión de Agua del Estado de México (CAEM)) 
Análisis de los factores subyacentes constructores de vulnerabilidades ante riesgo de desastres en el Estado de México

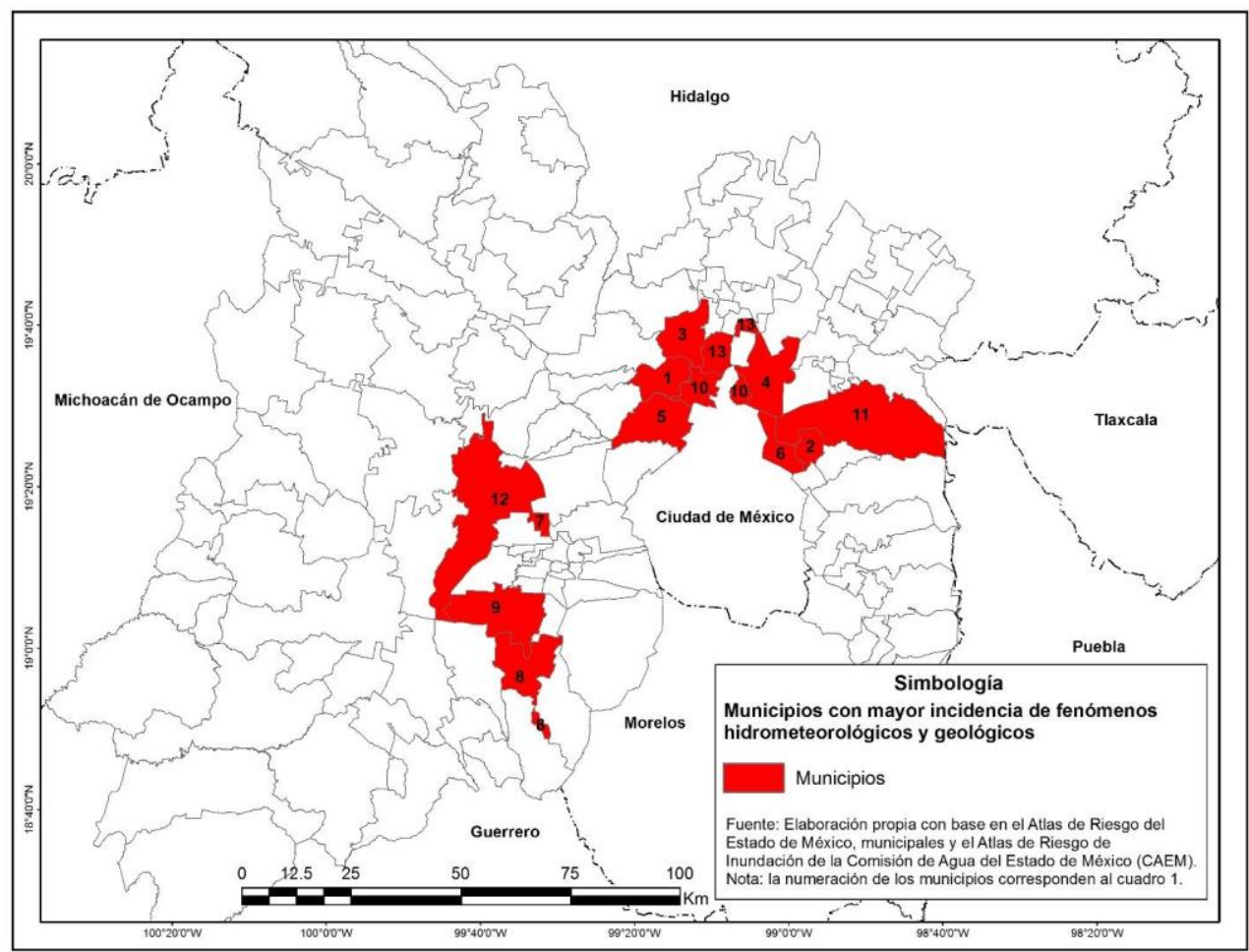

Mapa 1. Relación de municipios con mayor incidencia de fenómenos hidrometeorológicos y geológicos en el Estado de México

\begin{tabular}{|c|c|c|c|c|c|c|c|c|}
\hline \multirow[b]{2}{*}{ Municipio } & \multicolumn{2}{|c|}{ Período 2002 - 2010} & \multicolumn{6}{|c|}{ Período 2011 - 2016 (Población afectada -año) } \\
\hline & Reincidencia & $\begin{array}{c}\text { Población } \\
\text { afectada }\end{array}$ & 2011 & 2012 & 2013 & 2014 & 2015 & 2016 \\
\hline $\begin{array}{l}\text { Atizapan de } \\
\text { Zaragoza }\end{array}$ & 5 & 16490 & 3934 & 1869 & 1530 & 20 & 95 & 800 \\
\hline Chimalhuacán & 4 & 24775 & 542 & 9664 & 6510 & 30 & 300 & 120 \\
\hline $\begin{array}{l}\text { Coacalco de } \\
\text { Berriozábal }\end{array}$ & 5 & 8620 & 5490 & 1525 & 670 & 150 & 2500 & 1173 \\
\hline $\begin{array}{l}\text { Cuautitlán } \\
\text { Izcalli }\end{array}$ & 6 & 16366 & 2200 & 1626 & 3445 & 100 & 4266 & 1500 \\
\hline $\begin{array}{l}\text { Ecatepec de } \\
\text { Morelos }\end{array}$ & 8 & 50992 & 24746 & 13425 & 2995 & 745 & 1640 & 7045 \\
\hline Nezahualcóyotl & 8 & 38295 & 6294 & 11034 & 12235 & 2015 & 2025 & 6786 \\
\hline $\begin{array}{l}\text { Nicolás } \\
\text { Romero }\end{array}$ & 2 & 30 & 600 & 2850 & 1558 & 450 & 786 & 360 \\
\hline
\end{tabular}


José Emilio Baró Suárez - Armando Arriaga Rivera - Carlos Díaz Delgado

\begin{tabular}{l|cc|cccccc} 
Tlalnepantla de & 8 & 20410 & 1140 & 846 & 1960 & 1585 & ND & ND \\
Baz & 2 & 200 & 600 & 1836 & 1700 & ND & ND & ND \\
Tultepec & 8 & 19901 & 6258 & 1052 & 2927 & 50 & 1425 & 4875 \\
Tultitlán & & &
\end{tabular}

Cuadro 2. Principales afectaciones históricas por inundaciones en el Estado de México (Fuente: Atlas de Inundación de la CAEM, 2017)

\begin{tabular}{|c|c|c|c|c|}
\hline No. & Municipios & $\begin{array}{c}\text { Atlas de } \\
\text { Riesgo }\end{array}$ & $\begin{array}{c}\text { Programa de } \\
\text { Ordenamiento } \\
\text { Ecológico Municipal }\end{array}$ & $\begin{array}{c}\text { Programa de } \\
\text { Ordenamiento } \\
\text { Ecológico Regional } \\
\end{array}$ \\
\hline 1 & Acambay & 2014 & & \\
\hline 2 & Aculco & 2014 & & \\
\hline 3 & Almoloya de Juárez & 2016 & 2009 & 2003 \\
\hline 4 & Amanalco & & & 2003 \\
\hline 5 & Amecameca & 2011 & & 2007 \\
\hline 6 & Atenco & 2016 & & \\
\hline 7 & Atizapán de Zaragoza & 2015 & & \\
\hline 8 & Atlacomulco & 2013 & & \\
\hline 9 & Atlautla & 2015 & & 2007 \\
\hline 10 & Ayapango & & & 2007 \\
\hline 11 & Chalco & 2011 & & 2007 \\
\hline 12 & Chicoloapan & 2011 & & \\
\hline 13 & Chimalhuacán & 2011 & & \\
\hline 14 & Coatepec Harinas & & & 2011 \\
\hline 15 & Cocotitlán & & & 2007 \\
\hline 16 & Donato Guerra & & 2014 & 2003 \\
\hline 17 & Ecatepec de Morelos & 2013 & 2011 & \\
\hline 18 & Ecatzingo & & & 2007 \\
\hline 19 & El Oro & 2011 & & \\
\hline 20 & Huehuetoca & 2013 & 2012 & \\
\hline 21 & Ixtapaluca & 2018 & 2009 & 2007 \\
\hline 22 & Ixtapan de la Sal & 2013 & & 2011 \\
\hline 23 & Ixtapan del oro & & & \\
\hline 24 & Ixtlahuaca & 2015 & 2008 & \\
\hline 25 & Jilotepec & & 2013 & \\
\hline 26 & Jocotitlán & 2013 & & \\
\hline 27 & Juchitepec & 2014 & & 2007 \\
\hline 28 & $\mathrm{LaPaz}$ & 2014 & & \\
\hline 29 & Lerma & & & 2011 \\
\hline 30 & Metepec & 2013 & & 2011 \\
\hline 31 & Naucalpan de Juárez & 2010 & & \\
\hline 32 & Nezahualcóyotl & 2010 & & \\
\hline 33 & Nicolás Romero & 2011 & 2012 & \\
\hline 34 & Ocoyoacac & & & 2011 \\
\hline
\end{tabular}


Análisis de los factores subyacentes constructores de vulnerabilidades ante riesgo de desastres en el Estado de México

35 Ocuilan

36 Ozumba

37 Papalotla

2015

38 San Antonio la Isla 2016

39 San Felipe del Progreso 2014

40 San José del Rincón 2013

41 San Mateo Atenco 2017

42 Soyaniquilpan de Juárez 2014

43 Temamatla

2007

44 Temascalcingo

2013

45 Temascaltepec

46 Tenancingo

2012

47 Tenango del Valle 2013

48 Texcoco 2016

49 Teoloyucan 2014

50 Tepetlixpa

51 Texcaltitlán

52 Texcalyacac

53 Tlalmanalco

54 Tlalnepantla de Baz

55 Toluca

56 Tultitlán

57 Valle de Bravo

58 Villa de Allende

59 Villa del Carbón

60 Valle de Chalco

61 Villa Guerrero

62 Villa Victoria

2003

63 Xonacatlán

2011

64 Zinacantepec

2013

65 Zumpango

2014

2003 y 2011

Cuadro 3. Municipios del Estado de México con atlas de riesgo o algún instrumento de ordenamiento territorial, con su año de publicación (Fuente: Elaboración propia con base en gacetas de Gobierno de México, Atlas Municipales, SEDATU, 2018)

\begin{tabular}{lcc}
\hline \multicolumn{1}{c}{ Instrumento } & $\begin{array}{c}\text { Número de } \\
\text { municipios }\end{array}$ & $\begin{array}{c}\text { Porcentaje sobre el } \\
\text { total \% }\end{array}$ \\
\hline $\begin{array}{l}\text { Atlas de Riesgo } \\
\begin{array}{l}\text { Programa de ordenamiento ecológico } \\
\text { municipal }\end{array}\end{array}$ & 40 & 32 \\
$\begin{array}{l}\text { Programa de ordenamiento ecológico } \\
\text { regional (por municipio) }\end{array}$ & 13 & 10.4 \\
\hline
\end{tabular}


José Emilio Baró Suárez - Armando Arriaga Rivera - Carlos Díaz Delgado

Cuadro 4. Número de Atlas de Riesgo y Ordenamiento Territorial a nivel municipal (total: 125 municipios en el Estado de México) (Fuente: Elaboración propia con base en gacetas de Gobierno de México, Atlas Municipales, SEDATU, 2018)

\subsection{Desglose de factores subyacentes del riesgo}

Los factores identificados para evitar la generación y acumulación del riesgo fueron:

- Planificación y gestión deficiente del desarrollo urbano. Desarrollo inmobiliario incontrolado, inadecuados en materia de los usos del suelo o de las características geotécnicas de los materiales de soporte constructivos y deficiente diseño de las infraestructuras de drenaje y alcantarillado, además de su inadecuado mantenimiento.

- Escaso monitoreo y seguimiento del comportamiento geotécnico de las estructuras de contención de escurrimientos (bordos, canales, y presas de gavión, entre otras).

- Falta de mantenimiento de la infraestructura urbana de los drenajes y alcantarillado

- Planificación del desarrollo urbano en articulación con la construcción y ampliación de infraestructura de drenajes y alcantarillado para la evacuación de las escorrentías generadas por las grandes superficies impermeabilizadas resultado del crecimiento urbano de alta densidad.

- Especulación en los usos del suelo y con ello la falta de cumplimiento de normas y reglamentos.

- Carencia de una gestión de los ordenamientos territoriales y áreas naturales protegidas.

- Débil o ausente gestión de los Atlas de Riesgo por la existencia de lagunas legales, por un lado, y, por otro, por la escasa preparación y capacitación de los recursos humanos.

- Ausencia de una articulación entre los atlas de riesgo local, los ordenamientos territoriales, planes de desarrollo urbano (obras públicas) y regidurías ambientales municipales.

- Desconocimiento del marco legal en la Gestión Integral del Riesgo.

- Escasa capacidad recaudatoria de los municipios, lo cual dificulta la disponibilidad de recursos para la elaboración, actualización y seguimiento de los Atlas de Riesgos.

- Insuficiente cultura preventiva ante los riesgos naturales y también ausencia de seguros para cubrir estos riesgos. Solamente entre el 3\% y el 5\% de la población mexicana que viven bajo escenarios de riesgo se encuentra asegurada.

- Ausencia de una cultura más agresiva en la instrumentación y seguimiento de los sistemas de alerta temprana (SAT). Débil o ausente capacitación, superación y conocimiento de estos sistemas por parte de los recursos humanos de protección civil en los ámbitos locales.

- Desconocimiento de los bienes y servicios ambientales que prestan los diferentes ecosistemas, los cuales se encuentran presionados por fuerzas de cambios que generan escenarios de riesgo (enfoque ecosistémico en la Gestión de Riesgo). Adicionalmente, este desconocimiento trae como consecuencias la degradación ambiental. 
Análisis de los factores subyacentes constructores de vulnerabilidades ante riesgo de desastres en el Estado de México

- La deforestación en las zonas altas de las cuencas y subcuencas que canalizan caudales de escorrentías con grandes cargas de sedimentos, basuras y sólidos disueltos, mayormente contaminados, lo que cataliza también un desastre en materia de contaminación.

- La pobreza y la desigualdad (segregación urbana.) generan y acumulan un mayor riesgo de desastres en comunidades y hogares de bajos ingresos.

- Presencia muy limitada de infraestructura de investigación encaminada a la evaluación, sensibilización, comunicación y percepción del riesgo que contribuyan a la internalización de la gestión integral de riesgo.

\subsection{Factores subyacentes preponderantes constructores de escenarios de riesgo por inundación}

En este apartado se presentan los principales municipios del Estado de México que han sido afectados por las inundaciones y se describen los factores preponderantes constructores en cada caso (CAEM, 2017, DGCP s/f):

Municipio de Valle de Chalco Solidaridad

- En el municipio de Valle de Chalco transcurren tres corrientes: el río de la Compañía, el río Amecameca y el río Acapol (canal General), los cuales han sido utilizados como canales de aguas residuales. El río de la Compañía se encarga de conducir las aguas residuales provenientes de los municipios de Tlalmanalco e Ixtapaluca; el río Amecameca, drena la parte sur del municipio, y en esta región su caudal es utilizando para el riego de cultivos; y el río Acapol que drena la parte poniente del municipio. Este municipio se encuentra localizado en una de las partes más bajas de la zona Oriente del Estado de México, motivo por el cual se generan lagunas, en el límite con el municipio de Tláhuac, procedentes de los escurrimientos del cerro el Xico. En época de lluvias es necesario el bombeo de las aguas pluviales, para evitar inundaciones en la zona, utilizando los ríos antes mencionados como canales de desagüe; sin embargo, la cantidad de agua proveniente de las partes altas es cada vez mayor, saturando estos canales de desagüe $(\mathrm{H}$. Ayuntamiento de Valle de Chalco, 2011: 24). Como ejemplo cabe citar, las inundaciones del 5 de febrero de 2010, ya que la ruptura de los diques del río de La Compañía ocasionó un desbordamiento que inundó 23 colonias de Valle de Chalco, así como también colonias del municipio de Ixtapaluca, entre las que cabe destacar las colonias: El Triunfo, San Isidro, Avándaro y Unión de Guadalupe; afectando a tres mil viviendas y 15 mil habitantes (DGPC, s/f: 148).

Municipios de Tlalnepantla y Atizapán de Zaragoza

- El 29 de agosto del 2011 se registró una fuerte lluvia que provocó la inundación de varias calles y casas en los municipios de Tlalnepantla y Atizapán de Zaragoza. 
José Emilio Baró Suárez - Armando Arriaga Rivera - Carlos Díaz Delgado

Algunas de las zonas más afectadas fueron Las Arboledas y Valle Dorado; en este último caso, alrededor de 180 casas resultaron inundadas. El nivel del agua alcanzó hasta medio metro. Debido a la precipitación el drenaje de la zona se saturó y se taponeó con el Emisor Poniente, el cual se liberó con la apertura de compuertas del río Tlalnepantla. Dos días después, el 31 de agosto, un torrente de agua y lodo invadió 360 residencias de Valle Dorado, Arboledas y Mayorazgos de Los Gigantes, donde cientos de vecinos sufrieron cuantiosos daños.

- El 6 de septiembre de 2009 se registró una falla en el Emisor Poniente en el municipio de Tlalnepantla. Este conducto forma parte de una de las tres salidas artificiales con las que cuenta el Valle de México y conduce todos los escurrimientos provenientes de la zona poniente de esta entidad. La zona del siniestro se localizó en el camellón de la avenida Paseo de las Aves, a la altura de la calle de Caracas, en la colonia Valle Dorado. Las causas que originaron su falla fue la precipitación pluvial que se registró durante más de 40 minutos. Por este incidente resultaron con daños materiales mil viviendas y cinco mil habitantes afectados. Esta lluvia también afectó algunas comunidades de Atizapán de Zaragoza principalmente el hospital Herrejón. La falta de mantenimiento de la infraestructura urbana de los drenajes y alcantarillado contribuyó también a estas numerosas afectaciones (DGPC, s/f: 148).

Municipios de Ecatepec y Nezahualcóyotl

- La zona oriente del Valle de México, donde se localizan los municipios de Ecatepec y Nezahualcóyotl, ha resultado sumamente afectada por las fuertes precipitaciones. El 30 de octubre de 2009 se registró una lluvia por más de tres horas sobre la Sierra de Guadalupe que propició que los cauces naturales de la zona se desbordaran arrastrando a su paso viviendas y vehículos.

- El 4 de febrero de 2010, el deslave del bordo del Río de Los Remedios, en una longitud aproximada de 20 metros, afectó a tres mil 300 viviendas y a 16 mil 500 habitantes de los municipios de Ecatepec y Nezahualcóyotl.

- El 1 de julio de 2017, una lluvia producto de la tormenta tropical Arlene, rompió el récord histórico de precipitaciones en el valle de México que era de 36 mililitros en un día en 1989, ya que se reportaron 48.8 mililitros, casi el doble de lo que el sistema de drenaje puede sostener. En total 20 mil familias resultaron afectadas en diversas colonias de los municipios de Ecatepec y Nezahualcóyotl, luego que el exceso de lluvias provocara el desbordamiento del río de los Remedios y el de Xochiaca (DGPC, s/f: 148 y 149).

Municipio de Chicoloapan

- El riesgo de inundación es muy alto en casi todas las zonas que se ubican en las márgenes de los dos principales y únicos ríos que pasan por la zona poblada de este municipio, el río Coatepec y el río Manzano. Estos ríos, en general, están debidamente canalizados y reforzados con bordos, pero la violencia con que descienden las aguas

Acta Hispanica, Hungría, Supplementum II: 215-229, 2020, ISSN: 1416-7263 |223 
Análisis de los factores subyacentes constructores de vulnerabilidades ante riesgo de desastres en el Estado de México

de la parte alta de la subcuenca del Lago de Texcoco ha sobrepasado, en algunas ocasiones, las obras de infraestructura hidráulica del municipio. Dentro de la cabecera municipal, son también susceptibles las áreas bajas, que, aunque no están cerca de los ríos pueden inundarse debido a que las aguas llegan hasta esos lugares y los anegan (DGPC, s/f: 149).

Municipio de Chimalhuacán

- Las características físicas de los suelos en el municipio han provocado un desnivel gradual del terreno, que en épocas de lluvias ocasionan inundaciones estacionales, temporales y extraordinarias; este fenómeno se agrava por la ubicación de los drenes Chimalhuacán II y Acuitlapilco que cruzan al municipio, por lo que cuando la cantidad de agua pluvial que requiere ser expulsada es muy elevada se produce una sobresaturación de los drenes, sobrepasando los bordos artificiales. Por esta razón, las colonias localizadas en la cercanía con los drenes se ven continuamente afectadas, destacando los años 2009 y 2010 (H. Ayuntamiento de Chimalhuacán, 2011: 71).

Municipio de Juchitepec

- En 2010, los encharcamientos que se produjeron derivaron de unas intensas lluvias que provocaron escurrimientos en la zona urbana del municipio, así como también por el desbordamiento de una corriente intermitente que se localiza al poniente de la localidad. Estos encharcamientos se han reducido gracias a la ayuda de un cárcamo que se instaló en la zona de encharcamiento más baja, así se han reducido considerablemente los encharcamientos y las afectaciones a la población que se encuentra en la zona de riesgo asociada a este fenómeno (H. Ayuntamiento de Juchitepec, 2014: 261)

Municipio de Nezahualcóyotl

- Las inundaciones se presentan, de manera recurrente, en determinadas zonas durante los períodos de lluvias. Las medidas preventivas para evitar el desbordamiento de ríos o acumulación del agua en zonas bajas no son eficientes debido a la insuficiencia u obturación del drenaje por lo que deben ser monitoreadas antes de cada ciclo de lluvias. Los fenómenos identificados que pueden complicar una inundación en este municipio son: la acumulación de basura en el drenaje, la obturación del drenaje por sedimentos endurecidos y la caída de cenizas por alguna erupción del volcán Popocatépetl. Este volcán entró en una fase de mayor actividad entre 1919 y 1928; e inició otra fase similar en 1994, la cual se ha conservado hasta la actualidad (H. Ayuntamiento de Nezahualcóyotl, 2010: s/n).

Municipio de Texcoco

- Las localidades identificadas que son susceptibles a inundación son: Guadalupe Victoria, Palo Gacho, Wenceslao Victoria, Elsa Córdova, Paraje de Potrero Coatlichan, Lomas de Cristo, Lomas de San Esteban, Leyes de Reforma, Sector 
José Emilio Baró Suárez - Armando Arriaga Rivera - Carlos Díaz Delgado

popular y Tolimpa. En la temporada de lluvia, en la zona centro del municipio, se presentan encharcamientos prolongados que se deben a la falla del drenaje pluvial. Estos a su vez afectando al tránsito vehicular y algunas veces ocasiona accidentes vehiculares (H. Ayuntamiento de Texcoco, s/f: 71).

Municipio de San Mateo Atenco

- Este municipio es uno de los más afectados por inundaciones en el Estado de México. Las principales localidades que se ven afectadas por este fenómeno son diez, las cuales ya se encuentran totalmente identificadas, así como sus principales características como son nivel de agua originado por la inundación, la superficie afectada y la población vulnerable. En el cuadro 5 se detallan estas características para una inundación acaecida en el 2015 (H. Ayuntamiento de San Mateo Atenco, 2016: 134). La causa de las inundaciones en este municipio es fundamentalmente el desbordamiento del Río Lerma, el cual también forma parte del drenaje de aguas residuales del valle de Toluca. Además, el municipio se asienta en una zona deprima ya que en esta zona se ubicaba una de las lagunas que formó parte de un sistema lagunas, del cual aún quedan relictos en el valle (Lagunas de Almoloya del Río).

\begin{tabular}{lccc}
\hline Localidad & $\begin{array}{c}\text { Nivel de agua en } \\
\text { metros }\end{array}$ & $\begin{array}{c}\text { Superficie afectada } \\
\text { en hectáreas }\end{array}$ & $\begin{array}{c}\text { Número de población } \\
\text { damnificada }\end{array}$ \\
\hline Isidro Fabela & 1.50 & 115.90 & 511 \\
Santa María & 0.70 & 19.30 & 55 \\
San Isidro & 0.60 & 21.49 & 170 \\
La Concepción & 0.92 & 42.64 & 467 \\
San Pedro & 0.95 & 82.49 & 399 \\
San Juan & 0.95 & 70.80 & 219 \\
San Nicolás & 0.95 & 32.91 & 155 \\
Santiago & 1.00 & 25.87 & 183 \\
San Lucas & 1.00 & 35.94 & 229 \\
Guadalupe & 1.20 & 49.41 & 608 \\
\hline
\end{tabular}

Cuadro 5. Localidades vulnerables a las inundaciones localizadas en el municipio de San Mateo Atenco (Fuente: H. Ayuntamiento de San Mateo Atenco, 2016: 134)

Municipio de Zinacantepec

- Las inundaciones que se presentan en el municipio son principalmente de origen pluvial-fluvial (ribereñas y repentinas), es decir aquellas relacionadas con los ríos, los escurrimientos y sus cauces que son la "vía" por la que el agua precipitada recorre todo el municipio (H. Ayuntamiento de Zinacantepec, 2016: 85) 
Análisis de los factores subyacentes constructores de vulnerabilidades ante riesgo de desastres en el Estado de México

En resumen, los factores subyacentes que se presentan en los municipios del Estado de México afectados por las inundaciones se deben a la falta de saneamiento de los ríos y escurrimientos que bajan de las zonas altas inundando las vialidades y las viviendas de las localidades de las zonas bajas, haciendo que la infraestructura de drenaje sea insuficiente para captar grandes volúmenes de agua. Asimismo, en las zonas bajas, la red de drenaje se ve rebasada por las precipitaciones extraordinarias, que atascan y desbordan los canales de aguas residuales inundando las viviendas, causando grave daño al patrimonio de los mexiquenses.

\section{Propuestas de políticas públicas para la reducción de la vulnerabilidad ante riesgo de desastres en el Estado de México}

Las propuestas de políticas públicas para la reducción de la vulnerabilidad ante riesgo de desastres en el Estado de México corresponden a las siguientes acciones:

a) Acciones de gobernanzas urbana y local con base en la participación ciudadana en actividades de gestión y conocimiento del riesgo y en el fortalecimiento de las instituciones.

b) Creación de comités o consejos municipales (delegacionales) de gestión integral de riesgos con la participación voluntaria de actores sociales públicos, privados o de la sociedad en general.

c) Campañas de concientización y culturización de la población entorno a los principales peligros que la rodean, comenzando en las escuelas y centros laborales.

d) Internalización de la gestión integral del riesgo en el Plan Municipal de Desarrollo Urbano (PMDU), así como en los reglamentos de usos del suelo.

e) Verificación del cumplimiento de la normatividad constructiva o reglamentos de construcción.

f) Gestionar los Atlas de Riesgos y los de Ordenamientos Territorial con base en una gestión sistemática y transversal.

g) Comunicación social del riesgo local de desastres (radio, televisión, prensa).

h) Fortalecimiento de la resiliencia comunitaria y de la preparación, capacitación y superación del personal operativo y directivo de protección civil (Capítulo 8, Artículo 46, Ley General de Protección Civil).

i) Establecimiento, instrumentación y gestión continua del Sistema de Alerta Temprana (SAT). Este sistema también debe contemplar el monitoreo continuo de los procesos de hundimientos, subsidencias y agrietamientos del terreno. También es de gran valor el poder contemplar la realización de pronósticos con base en este monitoreo continuo.

j) Gestión del medio ambiente. Los servicios de los ecosistemas se pueden mejorar mediante una amplia gama de prácticas, entre las que se incluyen planificación integrada, zonas protegidas y pago por estos servicios. Todas estas prácticas mejoran 
José Emilio Baró Suárez - Armando Arriaga Rivera - Carlos Díaz Delgado

la regulación de las amenazas y los servicios de abastecimiento para comunidades urbanas y rurales.

k) Mecanismo de financiación del riesgo contando con el FONDEN (Fondo de Desastres Naturales) y FOPREDEN (Fondo para la Prevención de Desastres Naturales), y un conocimiento detallado de sus reglas de operación.

1) Los enfoques al nivel local y de la comunidad de la gestión de riesgos, en particular, cuando se sustentan en procesos efectivos de descentralización y en la asociación del gobierno con la sociedad civil, pueden potenciar la relevancia, efectividad y sostenibilidad de la reducción del riesgo de desastres en todos los sectores, así como reducir los costos y potenciar el capital social.

m) La prevención de desastres es un instrumento de planificación del desarrollo que permite intervenir las limitaciones impuestas por los eventos peligrosos y evitar que la propia acción del hombre produzca desastres. Las acciones y medidas de prevención, como instrumento de planificación, buscan intervenir en eventos que potencialmente pueden ocurrir con el fin de reducir al mínimo sus consecuencias, siendo necesario considerar las siguientes fases:

- Desarrollar el conocimiento sobre lo que puede pasar, es decir, sobre los riesgos existentes, lo que está expuesto a dicho peligro y, por consiguiente, lo que puede perderse en caso de manifestarse.

- Desarrollar los instrumentos de intervención necesarios, de acuerdo con el peligro existente, para evitar o reducir al mínimo los daños que este pueda causar.

- Para evitar las inundaciones en algunos casos se requiere de la construcción de diques con la finalidad de evitar inundaciones o la reubicación preventiva de viviendas. Asimismo, otras medidas preventivas de inundaciones es la limpia de las vías de comunicación y alcantarillado de residuos sólidos, para evitar inundaciones, en las zonas urbanas; monitoreo de las vías de comunicación en caso de inundación, para aplicar el plan de contingencia previamente establecido. Así como bordos en zonas rurales.

\section{Conclusiones}

Como conclusiones de este trabajo hay que destacar que solo el 52\% de los 125 municipios del Estado de México cuentan con algún instrumento de ordenamiento territorial o atlas de riesgos, y de estos el 32\% cuenta con atlas de riesgo municipal; además, y solo cuatro municipios cuentan con la actualización de su atlas, siendo: Ixtlahuaca (2017), Ecatepec de Morelos (2016), Tlalnepantla de Baz (2016) y Zinacantepec (2016).

Por un lado, falta casi el $48 \%$ de los municipios cuenten con su atlas de riesgos y, por el otro, se requieren las actualizaciones de los existentes. La falta de fechas o períodos para elaborarlos o actualizarlos, con base en la Ley, hace que los ayuntamientos no se vean obligados a realizarlos. Por si fuera poco, no existe presupuesto para la gestión, 
Análisis de los factores subyacentes constructores de vulnerabilidades ante riesgo de desastres en el Estado de México

ejecución, evaluación y actualización de los atlas de riesgos, lo cual permitiría determinar los alcances logrados y establecer lo que hace falta llevar a cabo por mitigar para prevenir desastres.

En los atlas de riesgos municipales existentes solo se consideraron las causas o factores subyacentes preponderantes con mayor afectación de riesgo socionatural por inundación en el caso de los municipios de Atizapán de Zaragoza, Chimalhuacán, Ecatepec de Morelos, Nezahualcóyotl, Tlalnepantla de Baz y Tultitlán.

Finalmente, con base en los diagnósticos municipales de riesgo se establecieron algunas propuestas de acción para proponer nuevas políticas públicas para la reducción de la vulnerabilidad ante riesgo en el Estado de México.

\section{Referencias bibliográficas}

Comisión del Agua del Estado de México (CAEM) (2017). Atlas de inundaciones. Gobierno del Estado de México.

Dirección de General de Protección Civil (DGPC) (s/f). Atlas de Riesgos del Estado de México. Gobierno del Estado de México.

Global Assessment Report (GAR) (2009). Global assessment report on disaster risk reduction. United Nations Office for Disaster Risk Reduction (UNDRR).

H. Ayuntamiento de Chimalhuacán (2011). Actualización del Atlas de Riesgos Naturales del Municipio de Chimalhuacán. H. Ayuntamiento de Chimalhuacán.

H. Ayuntamiento de Ecatepec de Morelos (2016). Actualización del Atlas de Riesgos del Municipio de Ecatepec de Morelos. Dirección de Protección Civil y Bomberos del Municipio de Ecatepec de Morelos.

H. Ayuntamiento de Ixtlahuaca (2017). Atlas de riesgos naturales del municipio de Ixtlabuaca, Estado de México. H. Ayuntamiento de Ixtlahuaca.

H. Ayuntamiento de Juchitepec (2014). Atlas de riesgos naturales del municipio de Juchitepec, Estado de México. H. Ayuntamiento de Juchitepec.

H. Ayuntamiento de Nezahualcóyotl (2010). Atlas de riesgos de Nezabualcóyotl. Protección Civil del H. Ayuntamiento de Nezahualcóyotl.

H. Ayuntamiento de San Mateo Atenco (2016). Atlas de riesgos. Etapa de actualización. Coordinación General de Protección Civil del H. Ayuntamiento de San Mateo Atenco.

H. Ayuntamiento de Texcoco (s/f), Atlas de riesgos del municipio de Texcoco. Dirección General de Protección Civil del H. Ayuntamiento de Texcoco.

H. Ayuntamiento de Tlalnepantla de Baz (2016). Atlas de Riesgo del municipio de Tlalnepantla de Baz. H. Ayuntamiento de Tlalnepantla de Baz.

H. Ayuntamiento de Valle de Chalco Solidaridad (2011). Atlas de Riesgo del municipio del $V$ alle de Chalco Solidaridad. H. Ayuntamiento de Valle de Chalco Solidaridad. 
José Emilio Baró Suárez - Armando Arriaga Rivera - Carlos Díaz Delgado

H. Ayuntamiento de Zinacantepec (2016). Atlas Municipal de Riesgos del municipio de Zinacantepec. Coordinación Municipal de Protección Civil y Bomberos del H. Ayuntamiento de Zinacantepec.

SEDATU (2018). Atlas Municipales de riesgos (SEDATU y elaborado por el municipio con recursos propios). Coordinación Nacional de Protección Civil y el Centro Nacional de Prevención de desastres (CENAPRED). Asequible en: http: //www.atlasnacionalderiesgos.gob.mx/ app/CoberturaMunicipal/, fecha de consulta: 05-09-2019. 